\title{
Contrôle des structures tourbillonnaires longitudinales dans le sillage d'une géométrie simplifiée de véhicule automobile : approche expérimentale
}

\author{
Benjamin Lehugeur ${ }^{\mathrm{a}}$, Patrick Gilliéron et Pierre Bobillier
}

Renault, Groupe Aérodynamique et Mécanique des Fluides, Technocentre, 1 Av. du Golf, 78288 Guyancourt Cedex, France

\begin{abstract}
Reçu le 31 mars 2008, accepté le 9 janvier 2009
Résumé - Une analyse du contrôle par soufflage des structures tourbillonnaires longitudinales qui prennent naissance sur les montants latéraux de lunette arrière d'un véhicule automobile est réalisée à partir d'une approche expérimentale sur le corps de Ahmed pour un angle de lunette arrière de $25^{\circ}$. Les mesures expérimentales s'effectuent à l'aide de vélocimétrie par imagerie de particules (PIV) stéréoscopique. Les résultats montrent que le soufflage est associé à une production de vorticité sur la partie supérieure de la lunette au niveau du cœur tourbillonnaire et une chute de la vorticité est relevée sur le bas de la lunette. Cette diminution de vorticité est de plus associée à l'apparition d'une zone de recirculation (vitesse longitudinale négative) dans le cœur tourbillonnaire caractéristique d'un éclatement tourbillonnaire. L'analyse des champs de vitesses azimutale et longitudinale relevés avec soufflage permet enfin de caractériser l'augmentation du nombre de Swirl du tourbillon longitudinal au-delà du seuil critique d'éclatement.
\end{abstract}

Mots clés : Aérodynamique / automobile / structure tourbillonnaire longitudinale / éclatement tourbillonnaire / contrôle / soufflage

\begin{abstract}
Control of longitudinal vortices in the wake of a simplified car geometry: experimental approach. The control by blowing techniques of longitudinal vortices originating at the rear pillars of a simplified automobile vehicle are analyzed by an experimental approach on the Ahmed body having a rear window angle of $25^{\circ}$. The experimental measurements are conducted according to stereoscopic Particle Image Velocimetry (Stereo-PIV). The results show that the blowing technique is associated to an increase of vorticity inside the vortex core on the upper part of the rear window and a decrease of vorticity on the lower part of the window. This vorticity drop is related to the onset of a recirculation zone (negative longitudinal velocities) in the vortex core which characterizes the vortex breakdown. The analysis of the azimuthal and longitudinal velocities allows to identify the increase of the Swirl number up to the critical point of vortex breakdown noted for high blowing velocities.
\end{abstract}

Key words: Aerodynamics / car / vortex / vortex breakdown / flow control / blowing

\section{Introduction}

En aérodynamique automobile, l'écoulement d'air au voisinage d'un véhicule automobile est caractérisé par l'apparition de décollements qui donnent naissance à des structures tourbillonnaires tridimensionnelles instationnaires. Parmi ces structures, les tourbillons longitudinaux localisés au niveau des montants de baie et des parties latérales de lunette arrière sont associés à des pertes d'énergie importantes [1,2] et sont responsables d'une partie non négligeable de la traînée aérodynamique [3].

\footnotetext{
a Auteur pour correspondance :

benjamin. lehugeur@airbus.com
}

Leur contribution dans la traînée d'un véhicule de type tri-corps peut atteindre $20 \%$ en régime autoroutier [4].

Dès lors, l'objectif consiste à rechercher des solutions nouvelles capables de supprimer ces structures, réduire leur développement dans le sillage et/ou provoquer leur éclatement. Le contrôle local du développement de ces structures peut également constituer une perspective intéressante pour améliorer la stabilité dynamique du véhicule lors des phases transitoires [5].

Les structures tourbillonnaires longitudinales appartiennent à la catégorie des écoulements de type jet/sillage tournant. Cette catégorie regroupe les tourbillons qui, en plus d'une composante de rotation, possèdent une composante de vitesse longitudinale. Les jets et les sillages 


\section{Nomenclature}

\begin{tabular}{|ll|}
\hline$d$ & Longueur de l'arête latérale de lunette arrière \\
$H$ & Hauteur de la géométrie simplifiée de véhicule automobile \\
$l$ & Largeur de la géométrie simplifiée de véhicule automobile \\
$L$ & Longueur de la géométrie simplifiée de véhicule automobile \\
$R e$ & Nombre de Reynolds \\
$S$ & Nombre de Swirl \\
$U_{\mathrm{x}}$ & Composante longitudinale du vecteur vitesse local \\
$U_{\theta}$ & Composante azimutale du vecteur vitesse local \\
$|V|$ & Amplitude de la vitesse locale \\
$V_{0}$ & Vitesse de l'écoulement infini amont \\
$\left(x_{0}, y_{0}, z_{0}\right)$ & Système de coordonnées associé à la lunette arrière \\
$(x, y, z)$ & Système de coordonnées associé au tourbillon longitudinal \\
$\alpha$ & Angle de l'axe tourbillonnaire par rapport au plan de la lunette arrière \\
$\beta$ & Angle de l'axe tourbillonnaire par rapport à la paroi latérale de la lunette \\
\hline
\end{tabular}

tournants constituent des systèmes instables qui peuvent, dans certaines conditions, disparaître par éclatement tourbillonnaire [6]. Lors de l'éclatement, la structure de ces systèmes évolue brutalement d'un état colonne quasiuniforme, à un état éclaté caractérisé par une zone de recirculation à laquelle est associée une remontée de pression statique. Ce processus constitue alors une solution pour réduire la contribution des culots de véhicules automobiles dans la traînée aérodynamique. Le travail présenté ici s'inscrit dans ce contexte. Plus généralement, l'éclatement tourbillonnaire est un phénomène spontané observé dans la nature, les tornades, les tourbillons d'apex d'ailes delta et de nombreuses configurations industrielles comme les chambres de combustion. En aérodynamique automobile, les structures tourbillonnaires longitudinales de lunette arrière n'éclatent pas spontanément. Dans cet article, l'objectif consiste à provoquer l'éclatement de ces structures à partir d'un soufflage distribué uniformément sur les parties latérales de lunette arrière. Ce soufflage est utilisé pour modifier la topologie de la structure tourbillonnaire, augmenter les pressions pariétales sur la lunette arrière et réduire les pertes d'énergie volumique dans le sillage.

Il s'agit aussi d'améliorer la connaissance des processus de formation, de transport et de dissipation des structures tourbillonnaires longitudinales ainsi que de valider des résultats numériques obtenus sur une géométrie identique, Lehugeur et al. [7-9]. Les résultats présentés dans cet article portent sur des expérimentations réalisées sur une géométrie simplifiée de corps de Ahmed [10] à partir de mesures de vitesse effectuées par PIV stéréoscopique. Le dispositif expérimental et les techniques de mesures sont présentés (Sect. 2) et les résultats obtenus analysés (Sect. 3).

\section{Moyens d'essais}

Le dispositif expérimental, les techniques de mesures et les configurations d'étude sont ici successivement décrits.

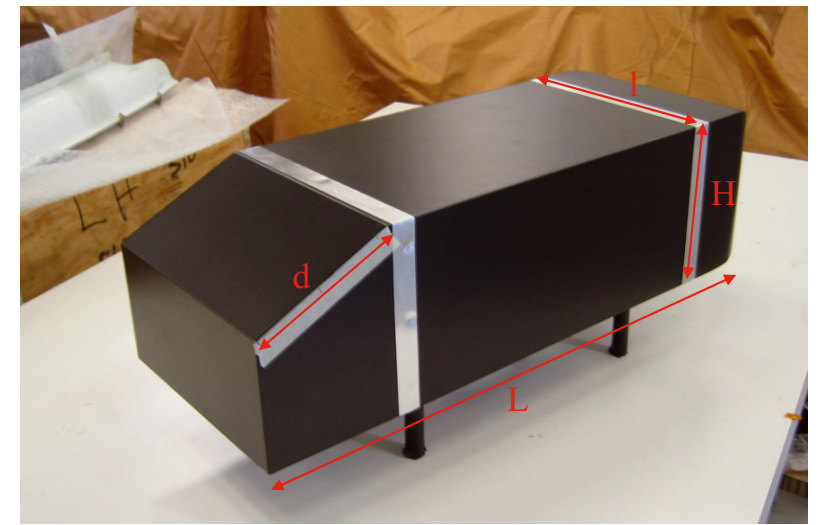

Fig. 1. Corps de Ahmed à l'échelle 0,7 et dimensions caractéristiques.

\subsection{Dispositif expérimental}

L'analyse s'effectue à partir d'une maquette en PVC et aluminium du corps de Ahmed à échelle 0,7 dont la longueur, la largeur et la hauteur, sont respectivement égales à $L=0,731 \mathrm{~m}, l=0,272 \mathrm{~m}, H=0,202 \mathrm{~m}$ (Fig. 1). La longueur de l'arête latérale de la lunette arrière $d$ est égale à $0,155 \mathrm{~m}$ et l'inclinaison de la lunette fait un angle de $25^{\circ}$ avec la direction de l'écoulement infini amont. Pour cette configuration, la structure décollée de lunette arrière est tridimensionnelle. Cette géométrie permet de reproduire une partie des phénomènes physiques qui apparaissent autour d'un véhicule automobile, [10]. Deux structures tourbillonnaires longitudinales contrarotatives prennent en particulier naissance au niveau des arêtes latérales de la lunette, se développent, s'évasent vers l'aval et sont dissipées dans le sillage.

Les essais sont réalisés dans la soufflerie Malavard du Laboratoire de Mécanique et d'Énergétique (LME) de l'École Supérieure de l'Énergie et des Matériaux d'Orléans (ESEM). Le corps de Ahmed est positionné au centre de la veine d'essais de section $2 \times 2 \mathrm{~m}^{2}$ et le rapport entre le maître couple $l \times H$ de la maquette et la surface transversale de la veine est voisin de $10^{-2}$. Les essais sont 

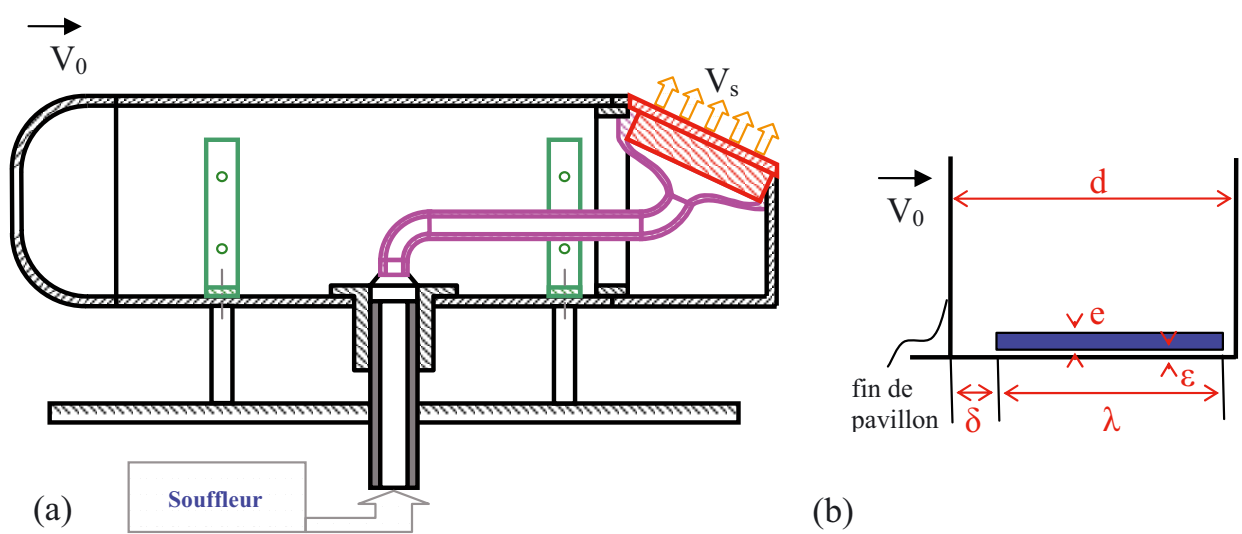

(b)

Fig. 2. (a) Corps de Ahmed avec dispositif de soufflage sur la lunette arrière, coupe suivant le plan médian longitudinal. (b) Fente de soufflage sur la lunette arrière, vue de dessus.

réalisés pour une vitesse $V_{0}$ de l'écoulement infini amont égale à $30 \mathrm{~m} . \mathrm{s}^{-1}$ et le nombre de Reynolds rapporté à la longueur de la maquette est voisin de $1,4 \times 10^{6}$. Le taux de turbulence de l'écoulement non perturbé par la présence de la maquette est proche de $0,4 \%$.

Le soufflage s'effectue au travers de deux orifices rectangulaires, positionnés à gauche et à droite de la lunette arrière, dont les géométries et positions sont définies à l'aide des longueurs caractéristiques $e, \varepsilon, \lambda$ et $\delta$ (Fig. 2b) respectivement égales à $1,4 \times 10^{-3} \mathrm{~m}, 1 \times 10^{-3} \mathrm{~m}$, $140 \times 10^{-3} \mathrm{~m}$ et $14 \times 10^{-3} \mathrm{~m}$. Le soufflage s'obtient à l'aide d'un souffleur (Fig. 2a) installé sous le plancher de la soufflerie dans une enceinte soumise à la pression atmosphérique et relié à la maquette par un tuyau souple. À l'intérieur de la maquette, le volume situé sous la lunette arrière et usiné en forme d'écope est relié aux fentes latérales de soufflage. Sa forme est optimisée pour limiter les pertes de charges et éviter les décollements parasites. Le souffleur possède un potentiomètre qui, en l'absence d'écoulement dans la veine d'essais, permet de faire varier la vitesse du jet $V_{\mathrm{s}}$ en sortie de fente (Fig. 2a) entre 7 et $70 \mathrm{~m} . \mathrm{s}^{-1}$. Les écarts de vitesse relevés par fil chaud, au niveau du plan longitudinal médian de la fente et à $10^{-3} \mathrm{~m} \mathrm{du}$ plan de lunette arrière dans la direction normale, n'excèdent alors pas $3 \%$. Lorsque le souffleur n'est pas actif, les orifices latéraux de lunette arrière restent ouverts pour simuler un écoulement d'habitacle. Dans ce cas et pour une vitesse d'air dans la veine d'essais égale à $30 \mathrm{~m} . \mathrm{s}^{-1}$, la vitesse de débit estimée de manière analytique au niveau des fentes de soufflage est proche de $10 \mathrm{~m} \cdot \mathrm{s}^{-1}$.

\subsection{Moyens de mesure}

Les champs de vitesse et de vorticité au voisinage de la lunette arrière s'obtiennent par PIV stéréoscopique. Cette technique non intrusive consiste à mesurer dans un même plan le déplacement de particules micrométriques transportées par l'écoulement à partir de deux caméras. Les caméras visualisent une même surface éclairée du plan laser suivant deux directions concourantes qui définissent deux angles aigus par rapport à la normale au plan laser. Ces deux angles sont fonctions de la place offerte au dispositif expérimental et relevés par calibration. La composition des trajectoires de particules vues par les deux caméras sur la même durée permet alors de déterminer les vitesses dans le plan et la normale au plan laser par simples projections géométriques.

La chaîne de mesure PIV stéréoscopique comprend un laser impulsionnel Nd-Yag à double cavité utilisé comme source lumineuse. Ce laser délivre des couples d'impulsions à une fréquence de $7 \mathrm{~Hz}$ avec une énergie de $190 \mathrm{~mJ}$ par impulsion centrée sur la longueur d'onde de $5,32 \times 10^{-1} \mu \mathrm{m}$. La durée d'une impulsion est égale à $10^{-8}$ ns et l'épaisseur du plan laser voisine de $2 \times 10^{-3} \mathrm{~m}$. Le temps $\Delta t$ entre deux impulsions successives, fixé par l'utilisateur, est voisin de $10^{-5} \mathrm{~s}$ et tel que les vitesses caractéristiques relevées dans le plan de mesure $\left(\sim 30 \mathrm{~m} . \mathrm{s}^{-1}\right)$ soient représentées par un déplacement qui n'excède pas 4 pixels sur l'image. La résolution spatiale est égale à $80 \mu \mathrm{m}$ par pixel et l'erreur relative de mesure proche de $4 \%$.

Les deux caméras utilisées possèdent une résolution de $2048 \times 2048$ pixels $^{2}$ et la cadence d'acquisition d'un couple d'image, égale à $10 \mathrm{~Hz}$, est synchronisée sur la fréquence du laser. Les opérations de calibration, d'enregistrement et d'extraction des champs de vitesse s'effectuent avec le logiciel INSIGHT $3 \mathrm{G}$ de TSI. Les images obtenues sont numérisées et enregistrées directement sur le disque dur d'un PC. Ce type d'acquisition, soumis aux fluctuations imposées par le système d'exploitation du PC, ne permet cependant pas d'assurer un pas de temps d'acquisition strictement constant et l'analyse fréquentielle des données instationnaires est impossible. Les résultats des différentes configurations présentées dans la suite du document sont alors moyennés sur 200 champs instantanés, pour un temps total d'acquisition voisin de $40 \mathrm{~s}$.

En pratique, la chaîne de mesure PIV est montée sur un bâti motorisé qui permet d'orienter aisément le laser à l'extérieur de la veine. Les caméras sont positionnées dans la veine sur un bras profilé (Fig. 3) et l'ensemencement de l'écoulement s'effectue à l'aide de particules d'huile d'olive. La région éclairée pour la mesure est recouverte 


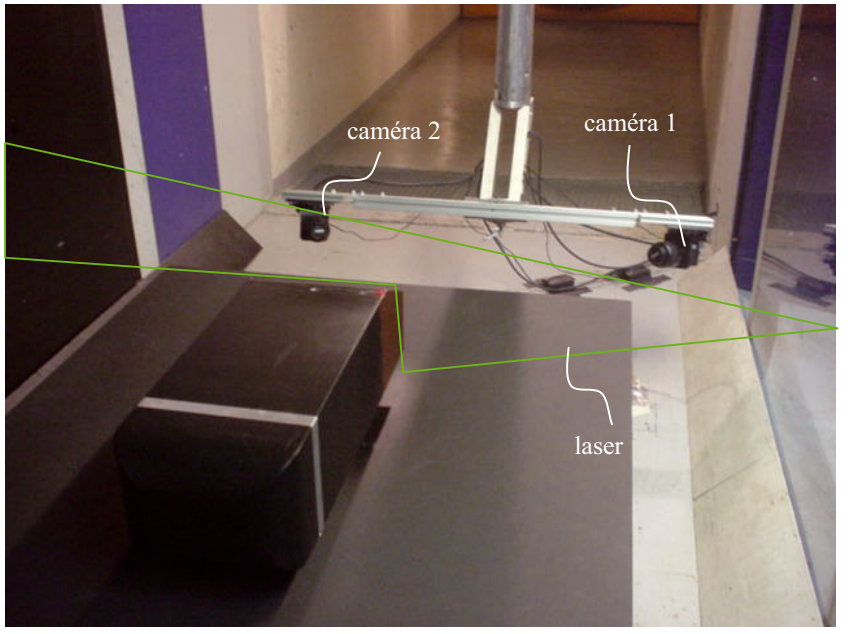

Fig. 3. Montage expérimental de PIV stéréoscopique.

d'un composé à base de rhodamine $\mathrm{B}$ et les caméras sont équipées d'un filtre interférentiel centré sur la fréquence du laser $\left(5,32 \times 10^{-1} \mu \mathrm{m}\right)$ à plus ou moins $0,05 \times 10^{-1} \mu \mathrm{m}$. Cette technique permet de réduire l'énergie lumineuse reçue par les caméras et l'intensité des réflexions lumineuses. La détermination des champs instantanés de vitesses PIV est enfin réalisée à partir de fenêtres d'interrogation de dimensions 16 pixels $\times 16$ pixels en utilisant un recouvrement de $50 \%$. Chaque champ PIV instantané est enfin traité à l'aide d'un algorithme de post-traitement qui supprime les vecteurs dont le rapport signal sur bruit (obtenu à partir du résultat du calcul d'inter-corrélation exprimé en niveau de gris) est inférieur à 1,2 .

\subsection{Configurations étudiées}

Compte tenu de la symétrie géométrique, l'analyse s'effectue sur la structure tourbillonnaire longitudinale gauche lorsque la maquette est vue de l'arrière. Le premier objectif consiste à identifier la position de l'axe tourbillonnaire à partir des extrema de vorticité relevés dans le cœur tourbillonnaire. Pour la géométrie considérée et des résultats expérimentaux [3,10] et numériques [2] obtenus sans contrôle, l'axe tourbillonnaire peut être considéré comme rectiligne et sa position stationnaire. La position de l'axe tourbillonnaire est alors définie à partir de mesures effectuées dans les plans P1 et P2 perpendiculaires au plan de la lunette arrière situés aux abscisses réduites $x_{0} / d=0,48$ et 0,88 , figure $4 \mathrm{a}$. La position de cet axe n'est pas recalculée lorsque le contrôle est appliqué.

Le second objectif consiste à analyser la topologie de la structure tourbillonnaire relevée avec et sans contrôle. L'axe tourbillonnaire sans contrôle étant défini dans le repère $\left(x_{0}, y_{0}, z_{0}\right)$ à partir des angles $\alpha$ et $\beta$ (Fig. 5), le plan laser et les deux caméras sont déplacés des mêmes angles et un nouveau repère d'étude $(x, y, z)$ est défini. Dans ce nouveau repère, l'axe des abscisses $x$ est aligné avec l'axe tourbillonnaire (Figs. 4b et 5) et la topologie du tourbillon est étudiée à partir de 7 plans de mesures parallèles distribués le long de l'axe tourbillonnaire aux abscisses réduites $x / d=0,19,0,32,0,45,0,58,0,71$, 0,83 et 0,97 . Dans chacun de ces 7 plans de dimensions $6 \times 10^{-2} \times 7 \times 10^{-2} \mathrm{~m}^{2}$, les mesures sont réalisées sans soufflage avec les fentes de soufflage ouvertes et pour les réglages du potentiomètre du souffleur correspondant aux vitesses $V_{\mathrm{s}}=0,5 V_{0}, V_{0}$ et $1,5 V_{0}$ relevées en l'absence d'écoulement dans la veine d'essais.

\section{Analyse des résultats}

L'analyse des champs moyens de vitesses PIV, obtenus à partir de 200 champs instantanés, est présentée dans les développements qui suivent. La position du tourbillon longitudinal est identifiée (Sect. 3.1) puis la topologie du tourbillon longitudinal, relevée sans et avec contrôle, est analysée au voisinage du cœur tourbillonnaire (Sect. 3.2).

\subsection{Identification du tourbillon longitudinal et changement de repère}

La position de l'axe tourbillonnaire est identifiée à partir des maxima de vorticité relevés dans les deux plans présentés dans la section 2.3 (voir Fig. 4a). L'axe tourbillonnaire est repéré par rapport au plan de la lunette arrière (angle $\alpha$, vue suivant la direction $z_{0}$, Fig. 5a), et par rapport à la paroi latérale adjacente de l'arrière du corps de Ahmed, (angle $\beta$, vue suivant la normale locale au plan de lunette arrière Fig. $5 \mathrm{~b}$ ). Les valeurs des angles $\alpha$ et $\beta$ sont respectivement voisines de $7^{\circ}$ et $5,5^{\circ}$. Ces valeurs sont identiques à celles obtenues numériquement par Lehugeur et al. [7] sur la même géométrie dépourvue de fente.

L'axe tourbillonnaire étant identifié, un nouveau repère d'étude $(x, y, z)$ est défini à partir du repère initial $\left(x_{0}, y_{0}, z_{0}\right)$ en effectuant deux rotations. Une première rotation d'angle $\alpha$ autour de l'axe $z_{0}$ transforme le repère initial $\left(x_{0}, y_{0}, z_{0}\right)$ en $\left(x_{1}, y_{1}, z_{1}\right)$ (Fig. 5a). Une deuxième rotation d'angle $\beta$ autour de l'axe $y_{1}$ transforme $\left(x_{1}, y_{1}\right.$, $z_{1}$ ) en $(x, y, z)$, (Fig. 5b). Les angles $\alpha \sim 7^{\circ}$ et $\beta \sim 5,5^{\circ}$ correspondent respectivement aux angles de précession et de nutation des angles d'Euler. L'axe $x$ est alors aligné avec l'axe de la structure tourbillonnaire longitudinale et l'origine du repère coïncide avec le coin supérieur gauche de la lunette arrière (vue de l'arrière). Les vitesses longitudinale $U_{x}$ et azimutale $U_{\theta}$ du tourbillon sont alors définies dans ce repère (Fig. 4b). En pratique, le plan laser est déplacé selon deux rotations d'angles $\alpha$ et $\beta$ puis translaté le long de la lunette arrière de bas en haut de la lunette arrière, pour obtenir les 7 plans de mesure présentés section 2.3 .

\subsection{Analyse de l'influence du contrôle}

La topologie du tourbillon longitudinal est analysée à partir des champs de vorticité, de vitesse longitudinale $U_{x}$ et du rapport $U_{\theta} / U_{x}$. Dans les développements qui suivent et pour chaque cartographie présentée, les positions de la 


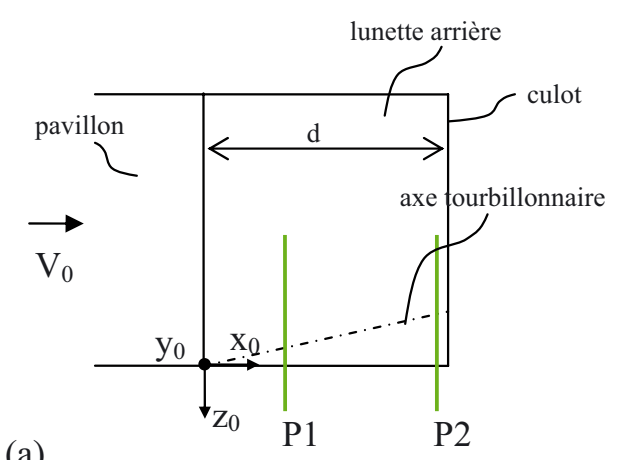

(a)

Fig. 4. (a) Définition des deux plans de mesures pour identifier l'axe tourbillonnaire, vue perpendiculaire à la lunette arrière. (b) Définition du repère associé au tourbillon longitudinal, vue de $3 / 4$ arrière gauche.

\section{(a)}

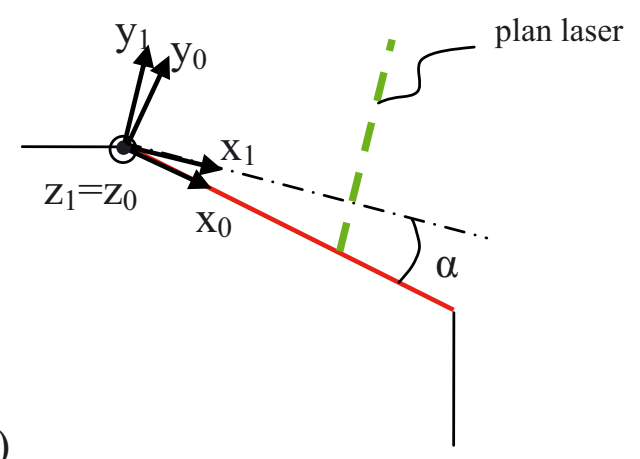

(b)

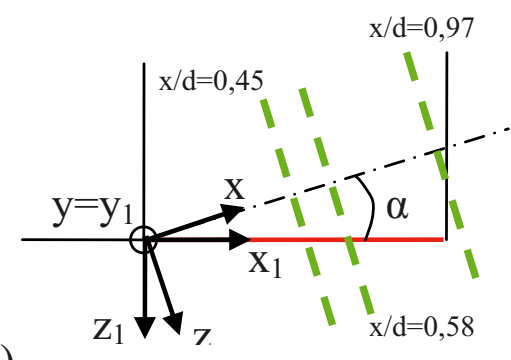

(a) Rotation d'angle $\alpha$, (b) rotation d'angle $\beta$. maquette (en noir) et de la fente de soufflage (en rouge) sont données à titre indicatif.

\subsubsection{Champs de vorticité}

La figure 6 représente des cartographies de vorticité longitudinale (composante suivant l'axe $x$ ) relevées à l'abscisse réduite $x / d=0,58$ pour les configurations sans et avec soufflage. Les résultats obtenus sans contrôle (Fig. 6) montrent que les minima de vorticité longitudinale (amplitude maximale pour des valeurs négatives), voisins de $-5000 \mathrm{~s}^{-1}$, sont relevés dans le cœur de la structure tourbillonnaire longitudinale de section quasicirculaire. La nappe décollée qui prend naissance au voisinage de l'arête latérale de la lunette, clairement identifiée figure 6 , est associée à des valeurs de vorticité longitudinale proches de $-3000 \mathrm{~s}^{-1}$. Cette nappe s'enroule autour de l'axe du tourbillon.

Le soufflage localisé au voisinage de l'arête latérale, associé à une production de vorticité négative à proximité de la fente, induit une diminution (augmentation en amplitude) des minima de vorticité autour de l'axe tourbillonnaire. Les valeurs de ces minima sont respectivement égales à $-5000 \mathrm{~s}^{-1},-5600 \mathrm{~s}^{-1}$ et $-7100 \mathrm{~s}^{-1}$ pour $V_{\mathrm{s}}=0,5 V_{0}, V_{0}$ et $1,5 V_{0}$. Ce résultat est semblable aux résultats numériques obtenus par Lehugeur et al. [9] sur la même géométrie. L'analyse met de plus en évidence un accroissement du domaine de vorticité positive à la périphérie de la structure tourbillonnaire. Cette évolution résulte de l'accroissement de la vitesse azimutale lorsque la vitesse de soufflage augmente, voir figure 6 .

L'influence du soufflage sur le champ de vorticité dans la structure tourbillonnaire est ensuite analysée sur le bas de la lunette arrière à l'abscisse réduite $x / d=0,97$ (Fig. 7). Sans contrôle, le coeur tourbillonnaire est associé à des vorticités longitudinales voisines de $-3200 \mathrm{~s}^{-1}$. Pour les configurations avec soufflage, les valeurs minimales de vorticité relevées dans la structure tourbillonnaire augmentent et sont respectivement proches de $-2800 \mathrm{~s}^{-1}$, $-2300 \mathrm{~s}^{-1}$ et $-2200 \mathrm{~s}^{-1}$ pour $V_{\mathrm{s}}=0,5 V_{0}, V_{0}$ et $1,5 V_{0}$. Cette diminution de vorticité (en amplitude), également identifiée par voie numérique $[8,9]$, suggère que le tourbillon longitudinal est déstructuré sur le bas de la lunette arrière.

\subsubsection{Champs de vitesse longitudinale}

Les figures 8 et 9 représentent les champs de vitesse longitudinale $U_{x}$ adimensionnée par la vitesse infinie amont $V_{0}$ aux abscisses relatives $x / d=0,58$ et 0,97 . Les résultats montrent que les vitesses longitudinales dans le cœur tourbillonnaire diminuent lorsque l'abscisse longitudinale augmente. Les plus faibles valeurs relevées sur l'axe tourbillonnaire aux abscisses réduites $x / d=0,58$ et 0,97 sont respectivement proches de $0,41 V_{0}$ et $0,27 V_{0}$. 

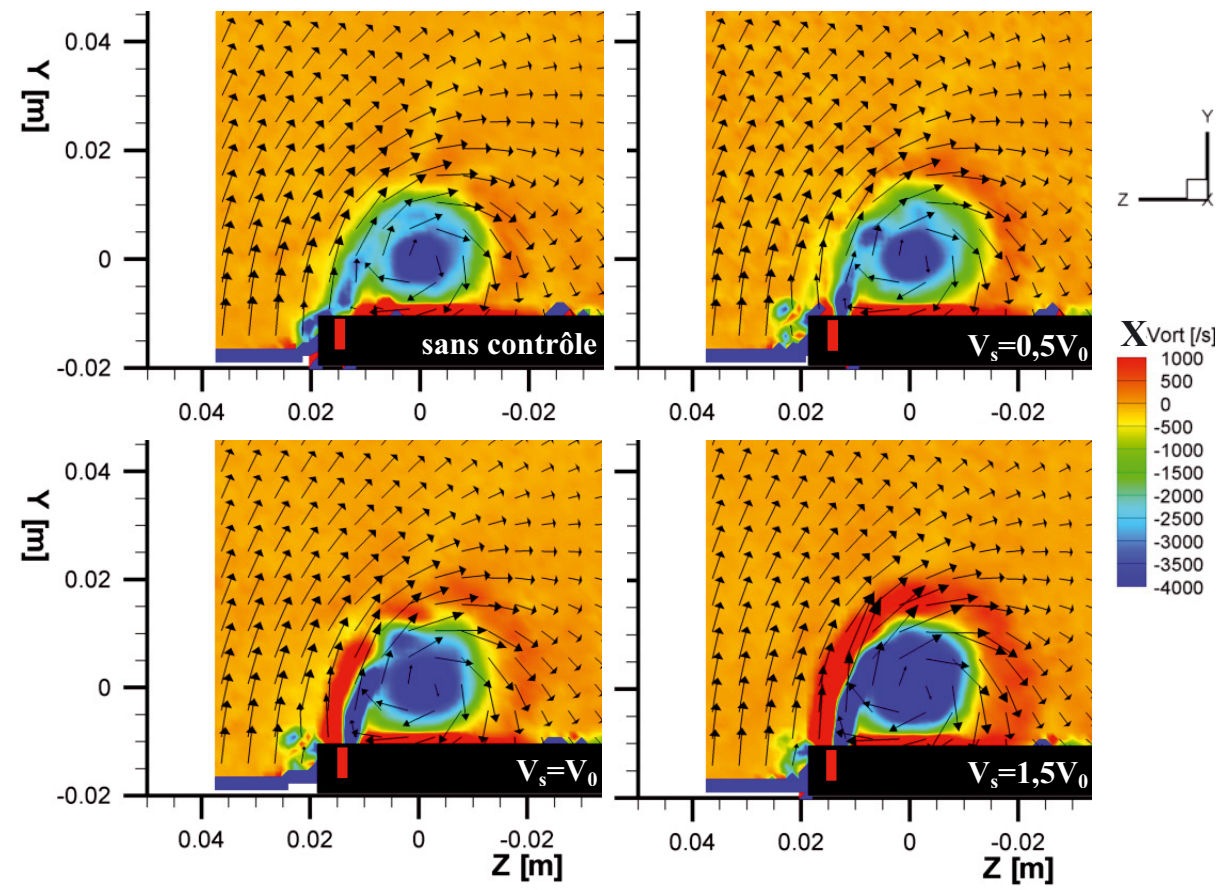

Fig. 6. Cartographies de la vorticité longitudinale relevée en $x / d=0,58$, pour les cas sans et avec soufflage pour les vitesses $V_{\mathrm{s}}=0,5 V_{0}, V_{0}, 1,5 V_{0}$.
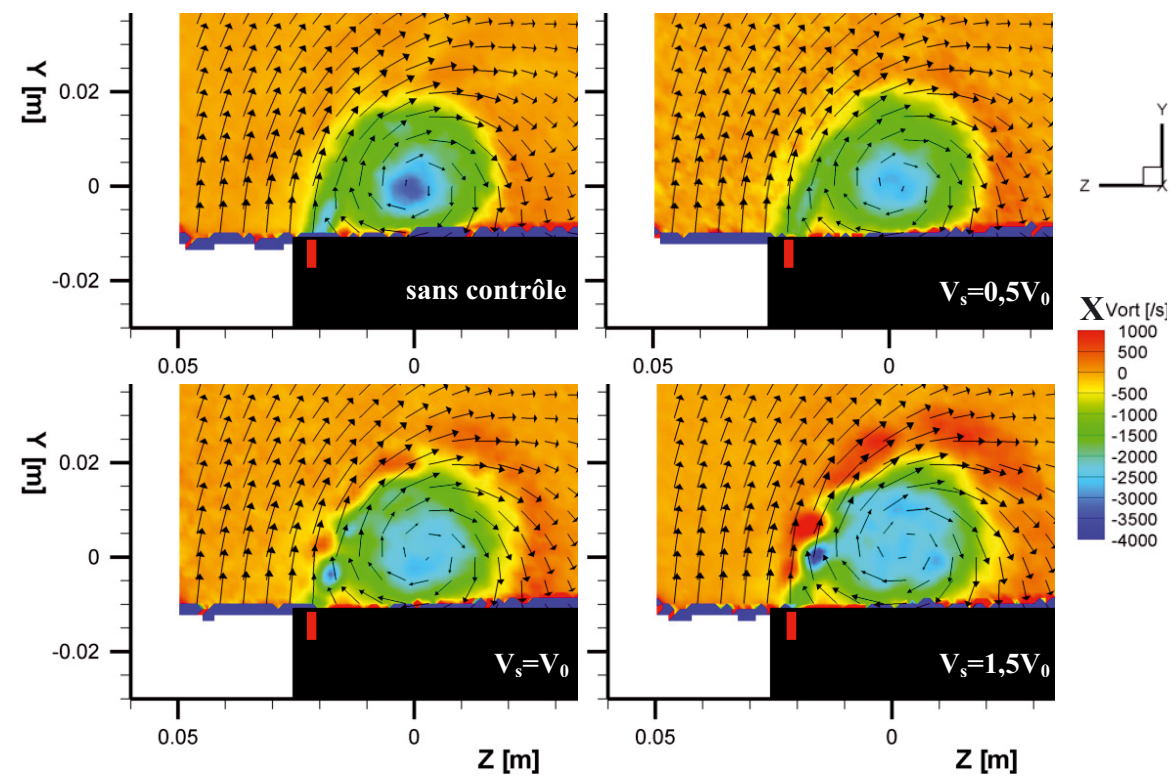

Fig. 7. Cartographies de la vorticité longitudinale relevée en $x / d=0,97$, pour les cas sans et avec soufflage pour les vitesses $V_{\mathrm{s}}=0,5 V_{0}, V_{0}, 1,5 V_{0}$.

Avec et sans contrôle, les vitesses longitudinales autour du centre tourbillonnaire sont inférieures à la vitesse de l'écoulement amont mais restent positives. Ce déficit de vitesse longitudinale dans le cœur tourbillonnaire montre que les tourbillons longitudinaux possèdent une structure de type sillage tournant, à l'inverse des résultats numériques et expérimentaux obtenus sur une maquette identique dépourvue de fente de soufflage $[7,11]$ (type jet tournant). Ce résultat, contradictoire avec le résultat obtenu avec des fentes latérales fermées, suggère alors une déstructuration induite par le soufflage naturel issu des fentes latérales restées ouvertes, (Fig. 2b).

Avec le soufflage, les minima de la vitesse longitudinale relative $U_{x} V_{0}^{-1}$ relevés dans le cour du tourbillon longitudinal diminuent jusqu'à tendre vers zéro pour $V_{\mathrm{s}}=$ $+1,5 V_{0}$, figures 8 et 9 . Cette réduction de vitesse longitudinale, également mise en évidence lors de l'éclatement d'un tourbillon d'apex d'aile delta [12], confirme l'hypothèse de la destructuration du cœur tourbillonnaire. 

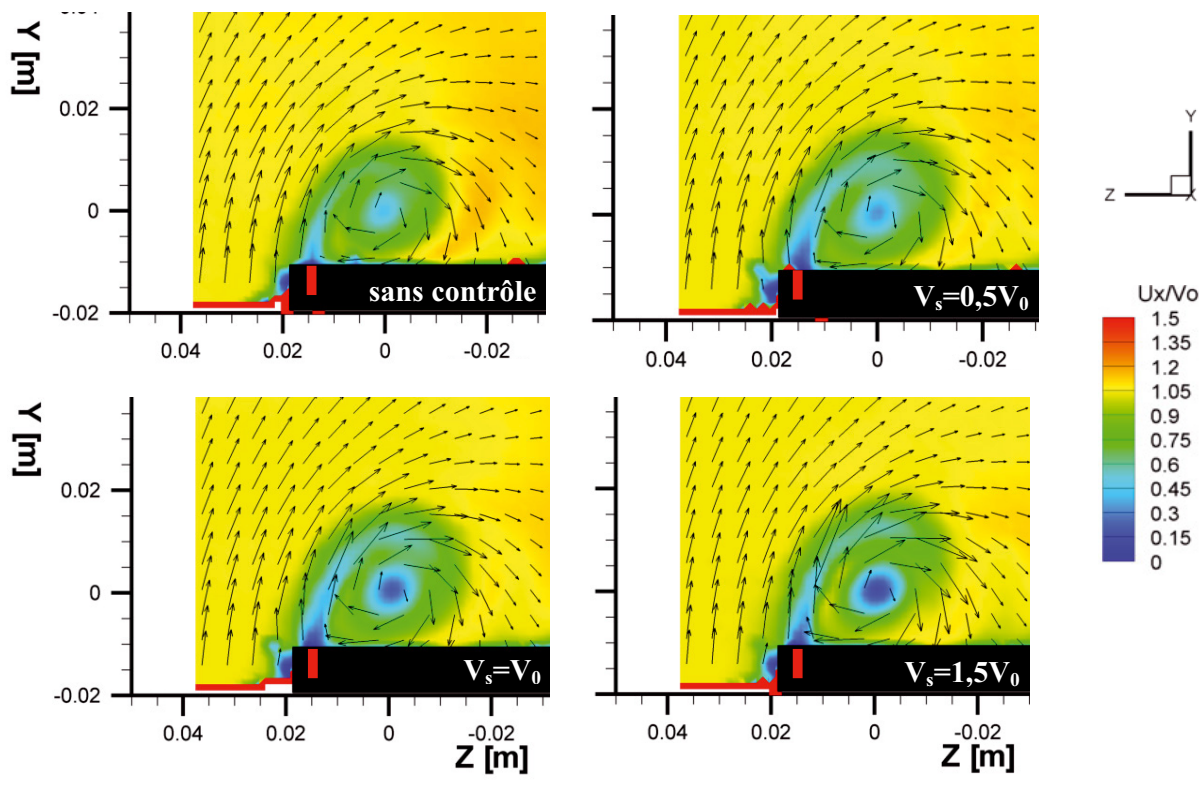

Fig. 8. Cartographies de la vitesse longitudinale relevée en $x / d=0,58$, pour les cas sans et avec soufflage pour les vitesses $V_{\mathrm{s}}=0,5 V_{0}, V_{0}, 1,5 V_{0}$.

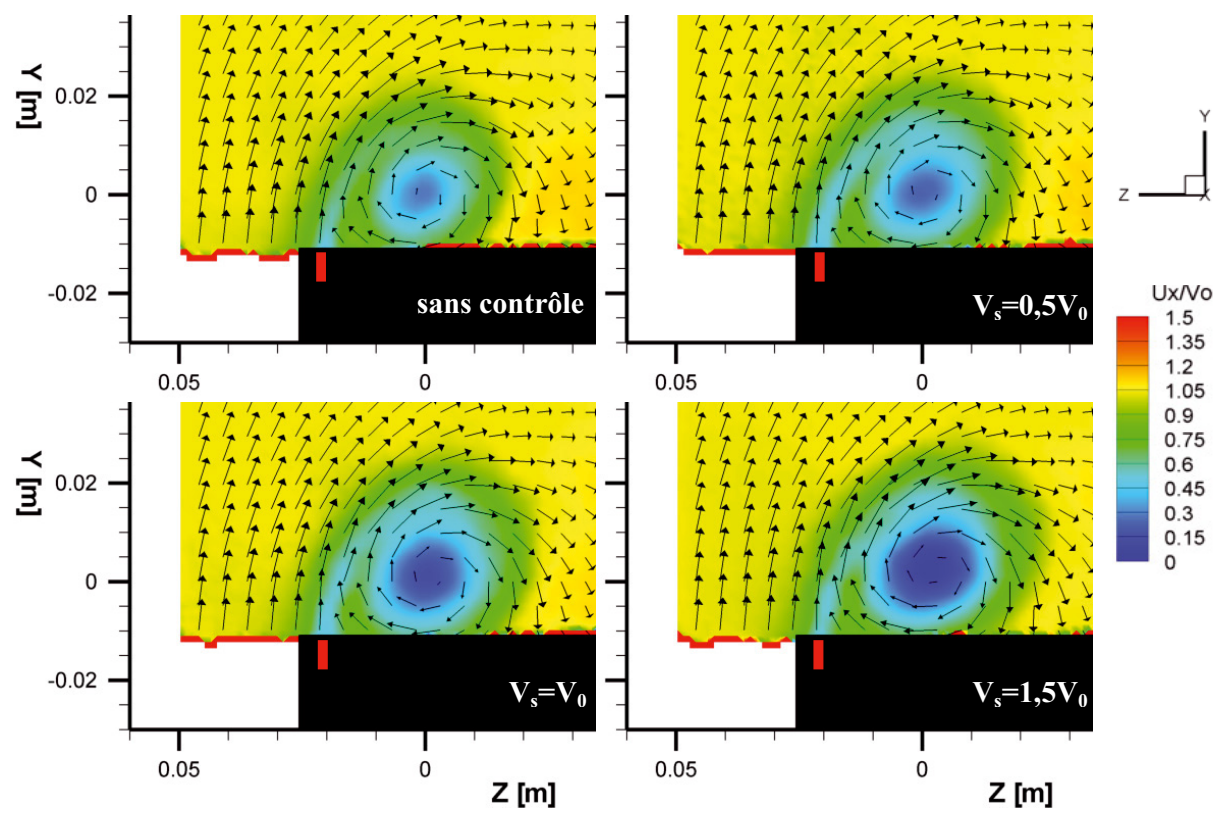

Fig. 9. Cartographies de la vitesse longitudinale relevée en $x / d=0,97$, pour les cas sans et avec soufflage pour les vitesses $V_{\mathrm{s}}=0,5 V_{0}, V_{0}, 1,5 V_{0}$.

L'analyse des champs instantanés de vitesses longitudinales (Fig. 10) met clairement en évidence un courant de retour dans le tourbillon longitudinal pour les valeurs négatives du rapport $U_{x} V_{0}^{-1}$ proches de $-0,2$. Ces valeurs sont associées à l'apparition d'une zone de recirculation $\left(U_{x} \leq 0\right)$ liée à l'existence d'un éclatement tourbillonnaire. Ce dernier phénomène étant instationnaire et associé à des évolutions spatiales rapides du champ de vitesse dans la zone de recirculation autour de l'axe tourbillonnaire, le champ de vitesse moyenné dans cette région tend vers zéro (Fig. 9). Les points blancs cerclés de rouge, repérés sur la figure 10, correspondent aux vecteurs vitesse éliminés lorsque le rapport signal/bruit devient inférieur à 1,2 .

\subsection{3 Éclatement tourbillonnaire}

Afin de mieux caractériser l'éclatement des structures tourbillonnaires de lunette arrière, l'influence du contrôle par soufflage est analysée à l'aide du nombre de Swirl $S$, défini à partir de la valeur maximale du rapport entre les 

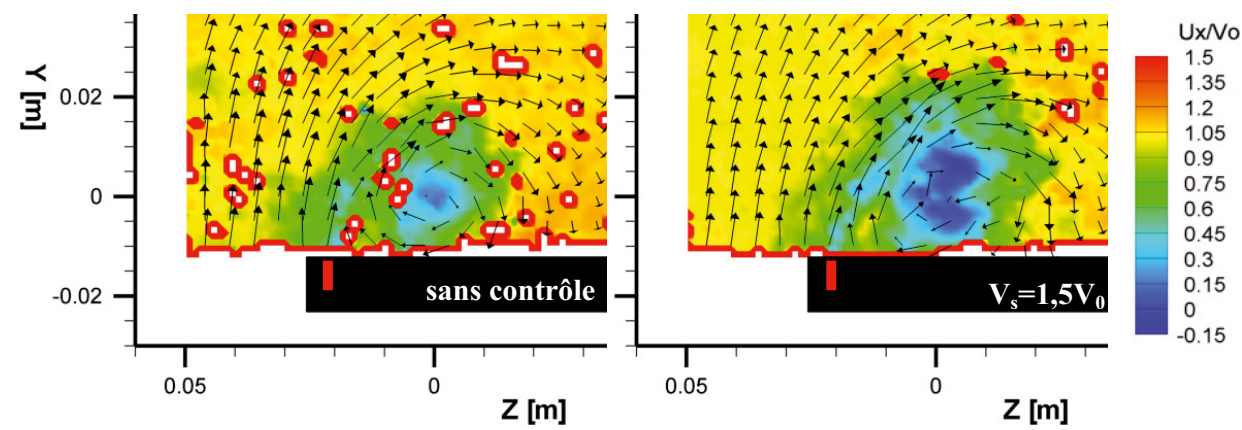

Fig. 10. Cartographies instantanées de la vitesse longitudinale relevée en $x / d=0,97$, pour le cas sans et avec soufflage pour les vitesse $V_{\mathrm{s}}=1,5 V_{0}$.

vitesses de rotation (vitesse azimutale $U_{\theta}$ ) et d'advection (vitesse longitudinale $\left.U_{x}\right)[12-14]$ :

$$
S=\max \frac{U_{\theta}}{U_{x}}
$$

Pour ce rapport, les expérimentations effectuées sur des jets tournants libres [6] ou confinés en conduite cylindrique [13] montrent que l'éclatement tourbillonnaire peut apparaître lorsque la valeur du nombre de Swirl $S$ dépasse la valeur critique $S_{\mathrm{c}}=1,5$. Ce résultat, confirmé analytiquement [6] et numériquement [15] constitue une condition nécessaire mais non suffisante d'éclatement. L'éclatement du jet tournant se traduit alors par l'apparition d'une zone de recirculation $\left(U_{x}<0\right)$ située en aval d'un point d'arrêt positionné au voisinage de l'axe tourbillonnaire.

Dans les cas classiques d'étude d'éclatement des jets tournants $[6,14]$, le nombre de Swirl est calculé à partir de profils de vitesse relevés à la sortie de l'orifice générant le jet tournant. Dans la présente étude, les profils de vitesse utilisés pour calculer le nombre de Swirl du tourbillon longitudinal de lunette arrière sont relevés à la plus petite abscisse pour laquelle le tourbillon possède une structure de type colonne identifiée à partir des champs de vitesse relevés par PIV. Cette position correspond à l'abscisse réduite $x / d=0,45$.

Les résultats reportés sur la figure 11 constituent une cartographie du rapport entre la vitesse azimutale $U_{\theta}$ et la vitesse longitudinale $U_{x}$ relevée à l'abscisse réduite $x / d=$ 0,45 .

Sans contrôle, la valeur maximale du rapport $U_{\theta} / U_{x}$, égale au nombre de Swirl et proche de 0,9, est relevée dans la nappe de vorticité qui s'enroule autour de l'axe tourbillonnaire. Ce résultat confirme la valeur de Swirl des tourbillons longitudinaux de lunette arrière, voisine de 1 , obtenue par voie numérique [8]. Au centre de la structure, le cour tourbillonnaire est visqueux, la vitesse azimutale et la valeur des rapports $U_{\theta} / U_{x}$ tendent vers zéro. Enfin, au voisinage de l'arête latérale de la lunette arrière, les faibles valeurs du rapport $U_{\theta} / U_{x}$, de l'ordre de 0,1 , sont associées à la présence du tourbillon secondaire contrarotatif identifié numériquement [2] sur une géométrie similaire.

Pour les trois configurations de soufflage, les maxima du rapport $U_{\theta} / U_{x}$, supérieurs à 1 , sont relevés au voisinage de la fente de soufflage (Fig. 11). Dans la structure tourbillonnaire, le nombre de Swirl $S$, est respectivement égal à $1,1,1,5$ et 1,6 pour les vitesses de soufflage $V_{\mathrm{s}}=0,5 V_{0}, V_{0}$ et $1,5 V_{0}$. Pour $V_{\mathrm{s}}=1,5 V_{0}$, la valeur du nombre de Swirl $(S=1,6)$ est alors à comparer au nombre de Swirl $(S \sim 1,5)$ obtenu pour l'éclatement d'un jet tournant libre [6]. Ce résultat confirme l'existence de l'éclatement tourbillonnaire mis en évidence pour cette vitesse $V_{\mathrm{s}}$ au paragraphe précédent à partir de l'analyse de vitesses longitudinales $U_{x}$.

\section{Conclusion}

L'influence du contrôle par soufflage sur la topologie d'une structure tourbillonnaire longitudinale est réalisée à partir d'expérimentations autour d'une géométrie simplifiée de type corps de Ahmed pour un angle de lunette arrière de 25 degrés. Les résultats s'obtiennent à l'aide de mesures de vitesses effectuées par PIV stéréoscopique. L'analyse des résultats valide et complète les résultats de simulations numériques [7-9] relevés sur une géométrie identique.

Les mesures de vitesse permettent de caractériser la structure du tourbillon longitudinal et la position angulaire moyenne de l'axe tourbillonnaire par rapport à la lunette arrière. L'axe tourbillonnaire étant identifié, l'évolution des champs de vitesses moyenne et instantanée est ensuite analysée dans le repère lié à la structure tourbillonnaire. Les résultats montrent que le soufflage est associé à une production de vorticité sur la partie supérieure de la lunette au niveau du cour tourbillonnaire et à une chute de vorticité sur le bas de la lunette. Cette diminution de vorticité est de plus associée à l'apparition d'une zone de recirculation (vitesse longitudinale négative) dans le cœur tourbillonnaire caractéristique d'un éclatement tourbillonnaire. L'analyse des champs de vitesses azimutale et longitudinale relevés avec soufflage permet enfin de caractériser l'augmentation du nombre de Swirl du tourbillon longitudinal au-delà $\mathrm{du}$ seuil critique d'éclatement $\left(V_{\mathrm{s}}=1,5 V_{0}\right)$ et mettre en évidence un éclatement tourbillonnaire pour la vitesse de soufflage $V_{\mathrm{s}}=1,5 V_{0}$. La prochaine étape consistera à améliorer le dispositif expérimental pour mesurer en soufflerie la traînée aérodynamique, valider les réductions de 


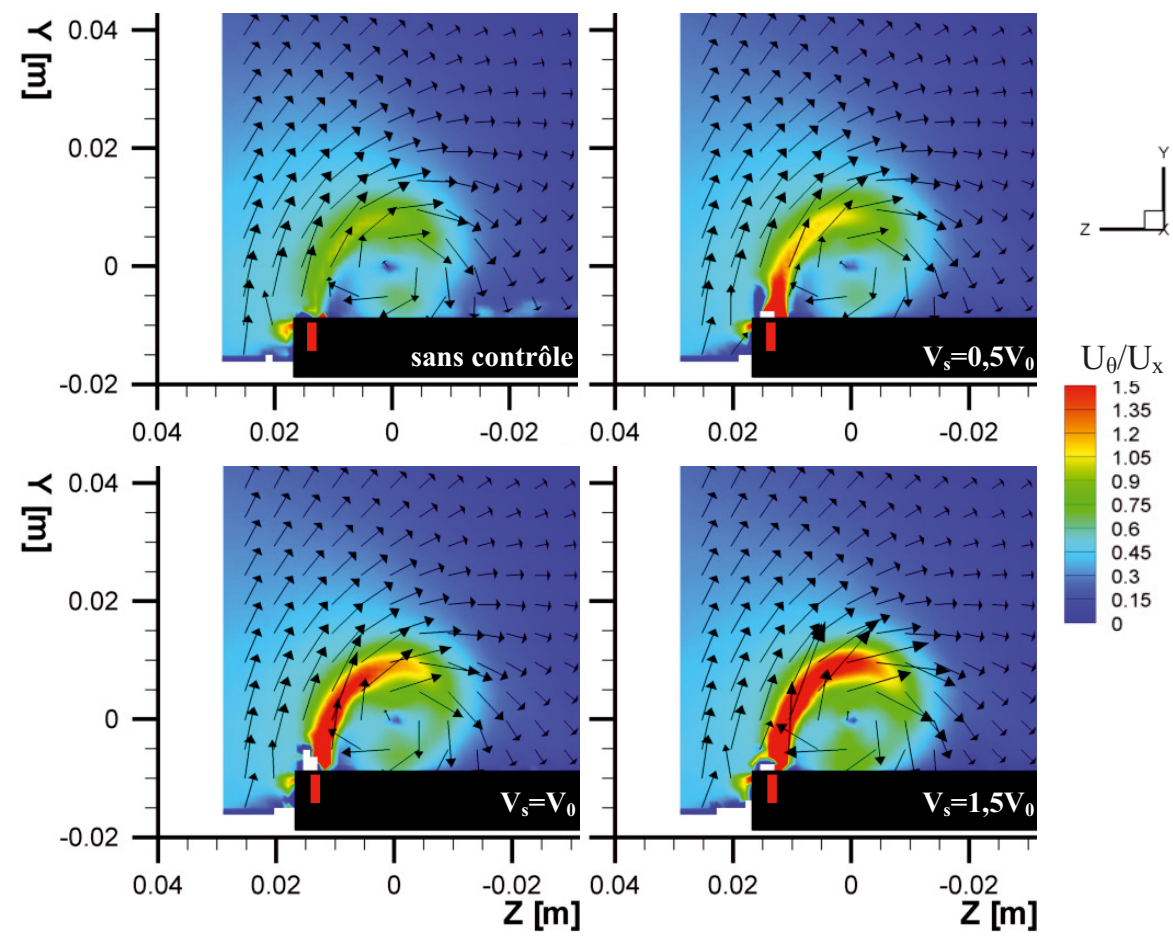

Fig. 11. Cartographies du rapport $U_{\theta} / U_{x}$ relevé en $x / d=0,45$, pour les cas sans et avec soufflage pour les vitesses $V_{\mathrm{s}}=0,5 V_{0}$, $V_{0}, 1,5 V_{0}$.

traînée de $6 \%$ obtenues par voie numérique [7-9] et accroître l'efficacité énergétique du contrôle.

Remerciements. Les auteurs souhaitent remercier Tanja Ivanic et le Laboratoire de Mécanique et d'Énergétique de l'École Supérieure de l'Énergie et des Matériaux d'Orléans pour leurs contributions à la réalisation des essais et au posttraitement des données PIV.

\section{Références}

[1] P. Gilliéron, J. Laurent, F. Chometon, Analyse des écoulements de culot par vélocimétrie laser, Application à l'automobile, $7^{\mathrm{e}}$ Congrès de vélocimétrie laser, 2000

[2] S. Krajnovic, L. Davidson, Contribution to large-Eddy simulation of the flow around a simplified car model, SAE International Congress \& Exposition, Detroit, Michigan, SAE 2004-01-0227, 2004

[3] J.F. Beaudoin, K. Gosse, O. Cadot, P. Paranthoen, B. Hamelin, M. Tissier, J.L. Aider, D. Allano, M. Gonzale, J.E. Weisfreid, Cavitation as a complementary tool for automotive aerodynamics, Experiments in Fluids 37 (2004) 763-768

[4] M. Onorato, A. Costelli, A. Garonne, Drag measurement through wake analysis, SAE International Congress \& Exposition, Detroit, Michigan, SAE SP-569, 1984

[5] P. Gilliéron, C. Noger, Contribution to the analysis of transient aerodynamic effects acting on vehicles, SAE International Congress \& Exposition, Detroit, Michigan, SAE 01-1311, 2004
[6] P. Billant, J.M. Chomaz, P. Huerre, Experimental study of vortex breakdown in swirling jets, J. Fluid Mech. 376 (1998) 183-219

[7] B. Lehugeur, Caractérisation et contrôle des structures tourbillonnaires longitudinales en aérodynamique automobile, Thèse de l'université Pierre et Marie Curie, 2007

[8] B. Lehugeur, P. Gilliéron, T. Ivanic, Contribution de l'éclatement tourbillonnaire à la réduction de la traînée des véhicules automobiles: approche numérique, Compte Rendus Mécanique, Éd. Elsevier 334, 2006, pp. 368-372

[9] B. Lehugeur, P. Gilliéron, Active control of vortex breakdown phenomenon in the wake of a simplified car geometry, ASME Joint U.S., European Fluids Engineering Summer Meeting, $N^{\circ}$ 2006-98349, July 17-20, Miami, FL, 2006

[10] S.R. Ahmed, R. Ramm, G. Falting, Some salient features of the time averaged ground vehicle wake, SAE technical paper series 840300, Detroit, 1984

[11] B. Lehugeur, P. Gilliéron, T. Ivanic, Characterization of C-pillar vortices by 3D particule image velocimetry, Proceedings of workshop on particle image velocimetry in car industry, Grugliasco, Italy, 2005

[12] F. Renac, Contrôle expérimental de l'écoulement tourbillonnaire sur une aile Delta, Thèse de doctorat, université de Paris VI, 2004

[13] J. Harvey, Some observations of the vortex breakdown phenomenon, J. Fluid Mech. 14 (1962) 585-592

[14] N.C. Lambourde, D.W. Bryer, The bursting of leadingedge vortices: some observations and discussion of the phenomenon, Aero. Res. Counc., Report \& Memoranda, $\mathrm{N}^{\circ}$ 3282, 1961, pp. 1-35

[15] M.R. Ruith, P. Chen, E. Meiburg, T. Maxworthy, Threedimensionnal vortex breakdown in swirling jets and wakes: DNS, J. Fluid Mech. 486 (2003) 331-378 